cases of generalised paralysis affecting the palate, pharynx, larynx, and diaphragm. But paralysed patients die from many other causes, thus differing from acute cases in which death is almost exclusively from heart failure. The most terrible cases of sudden death from syncope are those which occur when convalescence is established and the patient is thought to be free from danger. Death under these circumstances is always referable to some strain. Heart failure during convalescence (usually associated with paralysis), is shown by irregularity of pulse with or without signs of dilatation, and it is commonly determined by some strain, such as getting up too early, undue excitement, or vomiting; but the paralysis may be preceded by an irregular pulse, which may in turn have been determined by vomiting,

- From a cansideration of a number of cases Dr. Bolton says that the very great danger to which patients are liable during the whole course of diphtheria is at once apparent. This danger can only be rendered evident by a thorough and systematic examination of the heart and pulse in every case, however mild, and it can best be guarded against by keeping the patient in bed, or at least perfectly free from all excitement or strain as long as there are any signs of heart failure as shown by an irregular pulse or the physical signs of cardiac dilatation. During the acute stage the chief means of preventing cardiac failure consists in the use of efficient doses of antitoxin at as early a date as possible. Dr. Bolton points out that cardiac thrombosis, to which sudden deaths in diphtheria used to be attributed, is really a rare cause of death in that disease.

$$
1 \text { Edin. Med. Jour., April } 1902 .
$$

\section{PYREXIA IN THE PUERPERIUM.}

Dr. Ethel Williams ${ }^{1}$ draws attention to the considerable degrees of pyrexia which may occur during the puerperium and yet be produced not by uterine but by gastro-intestinal causes. The picture in most minds, when we speak of fever originating in the alimentary tract during the puerperium, is, 'she says, of a moderate fever of from $100^{\circ}$ to $102^{\circ}$ Fahr., occurring about the third day with little or no quickening of the pulse or constitutional disturbance-the whole thing vanishing as soon as the bowels are satisfactorily opened; and few people would feel happy in regarding as of purely gastrointestinal origin cases in which the temperature ran up to $103^{\circ}$ to $104^{\circ} \mathrm{Fahr}$, with a pulse of 120 to 140. She relates cases, however, which go to show that such may be the case, her object being to suggest that the rôle of the intestinal tube in infections during the puerperium is wider than it is generally supposed to be. It is known, she says, that during the puerperium the resistance of the body against microbic invasion is very markedly diminished, and although some of the cases referred to may be purely sapræmic, it is but natural to believe that this loss of defensive power will be as effective in allowing microbic invasion from the intestinal tube as from its more usual origin the genital tract. Unfortunately, Dr. Ethel Williams cannot find out any definite symptoms marking off these intestinal cases, unless one may rely upon the absence of such as point to the genital organs and the presence of Hatulence and pain in the upper quadrants of the abdomen. We may add, however, that the interesting observations which she has made may possibly go far to explain the striking relief which follows the use of saline aperients in some cases which, in default of such distinguishing signs, are often regarded as arising from sepsis in the genital tract ; for perhaps so-called puerperal sepsis may be more often intestinal than we imagine or at least may be so far complicated with intestinal sapræmia that by giving relief to that side of the trouble we may enable the patient to face her other difficulties with greater prospect of success.

1 Lancet, April 19.

\section{THE QUIESCENT PERIOD IN ACUTE} ABDOMINAL AFFECTIONS.

Ar the last meeting of the Medical Society a paper was read by Mr. A. H. Tubby on the Quiescent Period in the Course of Acute Abdominal Affections, in which he pointed out that after an acute onset in such cases there sometimes occurred a period of quiescence in which most of the symptoms might subside, the patient expressing himself as comfortable and free from pain; yet this might be but a prelude to an aggravation of the disease, with acute peritonitis and a fatal termination unless its significance were realised. This quiescent period was well marked in cases of injury to the intestines with or without rupture, intestinal perforation by ulceration, and other acute lesions, and particularly in acute appendicitis. The remission of symptoms was, however, never quite complete, and the importance of persistence of the altered proportion between pulse and temperature, of rigidity, of distension, and of vomiting was insisted upon. The main interest, however, both of Mr. Tubby's remarks and of the discussion which followed, lay in the support given to the important prognostic and indeed diagnostic significance of leucocytosis in abdominal affections. Leucocytosis was present in all these cases of latent mischief, and Mr. Tubby held that blood counts should not be omitted in any suspicious case. Leucocytosis was, he considered, of the utmost importance as an indication of the onset of acute peritonitis, and its presence in any marked degree afforded justifiable grounds for urging surgical interference without delay. Dr. J. H. Bryant also insisted upon the necessity of a blood examination in obscure abdominal cases where the question of operation arose, and he mentioned two cases, in one of which with a great excess of leucocytes a large abscess was found, while in the other in which there was but slight leucocytosis the inflammation subsided without suppuration. Mr. Jonathan Hutchinson, jun., on the other hand, thought that the evidence in regard to the value of leucocytosis as indicating operation was conflicting.

\section{LEUCOCYTOSIS IN APPENDICITIS.}

Is reference to this very important question as to the value of the indications given by the occurrence of leucocytosis, we cannot do better than refer to a recent article on leucocytosis as a point of prognosis in appendicitis by Dr. Henry M. Joy ${ }^{1}$ and Dr. Frederick T. Wright From a careful observation of a considerable number of cases they conclude that the degree of leucocytosis is an index of the severity of the case; a high 
stationary or an increasing leucocytosis indicates a progressively serious case which demands immediate intervention; a decreasing leucocytosis, save in some very few exceptional cases to be referred to again, points to a case of decreasing severity in which operation may be safely delayed, if for any reason it be so desired. It is interesting to observe that the authors fully recognise the danger of the quiescent period to which Mr. Tubby draws attention, for they say "Suppose, that in any given attack the usual signs and symptoms appear to indicate a marked diminution in its severity. If the first count be moderately high, say from 12,000 to 16,000 , and the second or any subsequent count show a rise of, say, 2,000 or more, especially if there be an apparently continuous rise, we urge immediate operation regardless of the fact that the clinical symptoms appear to show improvement. In such an instance we operate solely on the basis of the leucocyte count regardless of all other indications, and our experience, as we shall show in detail, seems to justify us in so doing." The exceptional cases in which, with a lowered leucocytosis, the patient may still be going to the bad, they explain by saying that " it is only (speaking somewhat loosely) when an effort is being made by Nature to overcome disease that we find an increasing leucocytosis, and per contra, when from an ever-increasing toxæmia Nature is forced to give up the struggle, there may be a progressive fall in the blood count." But, as they properly say, in such a case of appendicitis, seen at a late stage with a progressively-falling leucocytosis, the general conditions would be such that even the inexperienced could not be misled. Excluding these cases, however, the authors are quite clear as to the utility and reliability of the indications given by the blood count, which on the one hand may indicate a morbid condition of increasing severity which demands operation, no matter what the clinical symptoms may be, and on the other may show that operation may be postponed with safety should such a course, for other reasons, appear desirable.

1 New York Med. News, April 5.

\section{THE SUBCUTANEOUS INJECTION OF PARAFFIN IN THE TREATMENT OF SADDLE NOSE.}

WE have several times referred to the treatment of deformities due to loss or depression of the bones of the nose by the subcutaneous injection of melted paraffin, a proceeding which was we believe originated in Vienna. This method has now been applied in a fair number of cases in this country and with considerable success. A case of this sort was lately reported by Dr. Scanes Spicer. ${ }^{1}$ The patient, aged 25, had a well marked tip-tilted saddle nose and stunting of the nasal framework. She had suffered from childhood from nasal suppuration and fœtor. For the injection a 15-minim German glass hypodermic syringe was used, like that used for injecting tuberculin. Ten or twelve syringefuls of paraffin were injected under the skin, some downwards over the nasal bones, some upwards from the sides of the nose into the depressed gap, and the injected paraffin was moulded by an assistant's finger so as to shape the parts hefore setting. The points of injection were sealed up with collodion. The result so far as the appearance of the nose goes, is a very palpable bolstering up of the skin over the bony bridge of the nose and the formation of a very decent sized organ. The operation was performed ten months ago, and as the paraffin remains in the same state as when first injected, it may be regarded, says Dr. Spicer, as permanent, so far as we can see at present. Unfortunately in this case a small nodule of paraffin passed into the upper eyelid, from which it has not been possible to remove it, and there has been some œdema of the eyelids possibly from blockage of the lymphatic vessels by the paraffin. Dr. Downie Walker ${ }^{2}$ has also recorded a couple of cases in which this treatment was adopted with great success. The amount of paraffin injected was in one case a little over two drachms, and in the other "fully one drachm." While solidifying it was quickly moulded into the desired shape. "Sterilised paraffin," with a melting point of $104^{\circ} \mathrm{Fahr}$. was the material used, and the nose was prepared as for any other surgical operation. The paraffin in a molten condition was injected by means of a serum syringe, the nose being kept warm by hot dry sponge cloths, and the needle kept hot by an electric current. Pressure was applied around the nose and at the inner canthus of the eyes to confine the fluid to the nasal region.

1 Clinical Jour., A pril 9. 2 Brit. Med. Jour., May 3.

\section{AN EXAMPLE OF COMPULSORY}

\section{VACCINATION.}

Is October, 1898, small-pox was endemic in Porto Rico ; in December it was epidemic ; in January, 1899 , it had spread over the island, and by February there were over 3,000 recent cases. Under these circumstances the Americans having recently taken up "the white man's burden," determined to vaccinate the whole population. In February, the work was begun in all parts of the island, ${ }^{1}$ and was vigorously carried for four months, until by July 1st 860,000 vaccinations had been done in a population of about 960,000 persons. Of these $87 \frac{1}{2}$ per cent. were successful. The work then ceased; the disease had practically disappeared. In the two and a-half years that have since passed, instead of the former annual average death rate of 621 , the mortality from small-pox has been but two per annum. Here we have a good example of the grasp of principles and the thoroughness of method which are so characteristic of the inhabitants of the United States in all they undertake. The present position of vaccination in England is rapidly becoming as intolerable as it has long been inexcusable. If we spend enormous sums every year upon vaccination surely that is done in order that we may obtain protection from small-pox. Yet that object seems in practice to have been entirely put on one side, the trouble and the money which vaccination costs being so expended as to give in return the least possible protection. Truly we are a long-suffering and stupid people. For how many months have we not had this wretched small-pox wandering about London and the neighbourhood, and how many hundreds of thousands of pounds have we not already spent upon small-pox hospitals - a totally unnecessary and wasteful expenditure which we owe entirely ta the anti-vaccinists? The Amerizans 